\title{
Persistent currents in topological and trivial confinement in silicene
}

\author{
Piotr Jurkowski (1) and Bartłomiej Szafran (ㄷ) \\ AGH University of Science and Technology, Faculty of Physics and Applied Computer Science, al. Mickiewicza 30, 30-059 Kraków, Poland
}

(Received 1 November 2019; revised manuscript received 31 December 2019; accepted 21 January 2020; published 5 February 2020)

\begin{abstract}
We consider states bound at the flip of the electric field in buckled silicene. Along the electric flip lines a topological confinement is formed with the orientation of the charge current and the resulting magnetic dipole moment determined by the valley index. We compare the topological confinement to the trivial one that is due to a local reduction of the vertical electric field but without energy gap inversion. For the latter the valley does not protect the orientation of the magnetic dipole moment from inversion by external magnetic field. We demonstrate that the topologically confined states can couple and form extended bonding or antibonding orbitals with the energy splitting influenced by the geometry and the external magnetic field.
\end{abstract}

DOI: 10.1103/PhysRevB.101.075408

\section{INTRODUCTION}

A clean electrostatic confinement of charge carriers in quantum dots provides an environment for precise studies of localized states, energy spectra, coherence times [1], and electron-electron interactions [2], as well as for manipulation of the charge [3,4], spin [5], and valley [6] degrees of freedom. In gapless graphene the carrier confinement by electrostatic potentials is excluded by the Klein tunneling [7]. The electrostatic confinement becomes available when the energy gap is opened by a vertical electric field-in bilayer graphene [8-12] and in silicene [13,14]. Silicene is an atomic monolayer graphene-like material [15-19] with a buckled [15] crystal lattice. The spatial control of the energy gap by gating allows for electrostatic confinement of charge carriers and formation of quantum-dot bound states with discrete energy spectra; see Refs. [6,20-23] for bilayer graphene and Ref. [24] for silicene.

In both bilayer graphene and in silicene the energy gap can be locally inverted by the flip of the electric field vector. The flip of the vertical electric field forms a topological confinement of chiral currents along the zero line of the symmetry-breaking potential [25-28] with bands that appear within the energy gap. For bilayer graphene this confinement is also achieved at the stacking domain walls induced by line defects [29,30] or twist of the layers [31,32]. In silicene [27] and staggered monolayer graphene [28] the topological band is single and linear as a function of the wave vector while two nonlinear bands appear in bilayer graphene [25,26,28,29]. The reflectionless one-dimensional channels that appear with the flip of the electric field [25-28,33] are similar to the edge channels in the quantum Hall spin insulators [34-36], only with the valley degree of freedom replacing the spin in the protection mechanism against backscattering. Similar confinement of unidirectional currents in the bulk of the monolayer graphene is observed at $n-p$ junctions but only at strong magnetic fields [37-43].

Here, we consider quasi-zero-dimensional states localized along closed lines of the flip of the vertical electric field in buckled silicene. The states appear within a locally vanishing energy gap. We find that the chiral nature of the confined states is revealed by the direction of current circulation around the zero lines that is strictly related to the valley degree of freedom of the confined states. When the external magnetic field is applied the sign of the energy response depends only on the valley state. Similarly, the current in the topological confinement cannot be reoriented for a given state by the external magnetic field, unlike the persistent currents [44,45] for metals [46,47], semiconductors [48-50], or etched graphene [51-53] quantum rings. We compare the results for the topological confinement with the trivial one resulting from the spatial variation of the energy gap without the inversion of the conductance and valence bands. For the trivial confinement the external magnetic field can reorient the current. In this respect the loops of current at the trivial electrostatic confinement are similar to the ones flowing in etched graphene quantum rings [51-54]. We show that the topological confinement loops at separate zero lines form extended orbitals as in double quantum dots [55].

\section{THEORY}

We consider a buckled silicene monolayer in an inhomogeneous electric field. We consider first the system with a circular symmetry (see Fig. 1) with the potential bias between the sublattices that changes along the radial direction. We set the potential at the $A$ sublattice

$$
V_{A}(\mathbf{r})=V_{g}\left[1-2 \exp \left(-r^{4} / R^{4}\right)\right],
$$

and assume that for the silicene placed symmetrically between the gates the potential on the $B$ sublattice is opposite $V_{B}(\mathbf{r})=-V_{A}(\mathbf{r})$. For the potential given by Eq. (1) the electric field changes orientation at a distance $r=\ln (2)^{1 / 4} R$ from the origin. For the negative potential on the $A$ sublattice in the potential center the $K\left(K^{\prime}\right)$ the electron currents flow clockwise (counterclockwise) along the flip of the electric field [33]. 


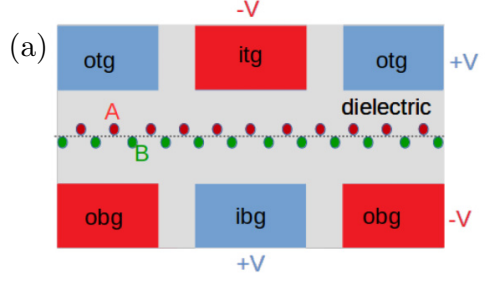

(b)

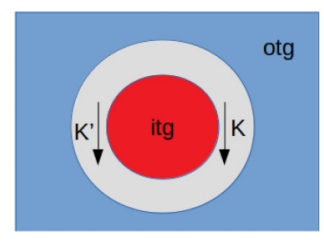

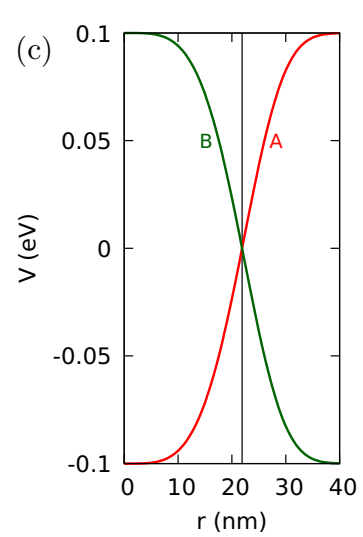

FIG. 1. (a) Side view of the considered system. A buckled silicene layer (green and red dots stand for the $\mathrm{Si}$ atoms at the $B$ and $A$ sublattices, respectively) is embedded within a dielectric (gray area) in a system of metal gates inducing negative (red) and positive (blue) potential energy for electrons. The sublattice $A(B)$ is closer to the top gates (bottom gates). "obg" and "ibg" ("otg" and "itg") stand for outer and inner bottom (top) gates. Panel (b) shows the top view of the system from the $A$-sublattice side. In (c) we plot the model potential on the $A$ (red) and $B$ (green) sublattices for the potential of a rotational symmetry [Eq. (1)] with $r$ standing for the distance from the origin, for $V_{g}=0.1 \mathrm{eV}$ and $R=24 \mathrm{~nm}$. The potential on the $B$ sublattice is opposite $V_{B}=-V_{A}$. The arrows in (b) show the direction of the valley-polarized electron currents for states confined at the electric field flip. The $K^{\prime}(K)$ electrons move with the negative potential on the $A$ sublattice on the left (right) hand side, i.e., counterclockwise (clockwise).

\section{A. Atomistic tight-binding Hamiltonian}

The states in both circular and lower symmetry potentials are also analyzed with the atomistic tight-binding Hamiltonian [17-19],

$$
\begin{aligned}
H_{\mathrm{TB}}= & -t \sum_{\langle k, l\rangle} p_{k l} c_{k}^{\dagger} c_{l}+i t_{\mathrm{SO}} \sigma_{z} \sum_{\langle\langle k, l\rangle\rangle} p_{k l} v_{k l} c_{k}^{\dagger} c_{l} \\
& +\sum_{k} V_{k} c_{k}^{\dagger} c_{k}+\frac{g \mu_{B} B}{2} \sigma_{z} .
\end{aligned}
$$

The first sum describes the nearest-neighbor hopping. The second sum is the atomistic form of the intrinsic spin-orbit interaction [56] with $v_{k l}= \pm 1$. The sign of $v_{k l}$ is positive (negative) for the next-nearest-neighbor hopping via the common neighbor ion that turns counterclockwise (clockwise) and $p_{k l}$ is the Peierls phase that introduces the magnetic field $p_{k l}=e^{i \frac{e}{\hbar} \int_{r_{k}}^{r_{l}} \overrightarrow{\mathbf{A}} \cdot \overrightarrow{d l} l}$, where $\mathbf{A}$ is the vector potential. We use the symmetric gauge $\mathbf{A}=(-B y / 2, B x / 2,0)$ for the magnetic field perpendicular to the silicene lattice $(0,0, B)$. The tight-binding nearest-neighbor hopping Hamiltonian is $t=$ $1.6 \mathrm{eV}[18,19]$, and $t_{\mathrm{SO}}=3.9 \mathrm{meV}$ is the intrinsic spin-orbit coupling constant $[18,19]$. The positions of the ions of the $A$ sublattice $\mathbf{r}_{\mathbf{k}}^{A}=k_{1} \mathbf{a}_{1}+k_{2} \mathbf{a}_{2}$ are generated with the crystal lattice vectors $\mathbf{a}_{1}=a\left(\frac{1}{2}, \frac{\sqrt{3}}{2}, 0\right)$ and $\mathbf{a}_{2}=a(1,0,0)$, where $a=$ $3.89 \AA$ is the silicene lattice constant, and $k_{1}, k_{2}$ are integers. The $B$ sublattice ions are generated by $\mathbf{r}_{\mathbf{k}}^{B}=\mathbf{r}_{\mathbf{k}}^{A}+(0, d, \delta)$, with the in-plane nearest-neighbor distance $d=2.25 \AA$, and the vertical shift of the sublattices $\delta=0.46 \AA$. In Eq. (2) $V_{k}$ is the electrostatic potential on ion $k$. The intrinsic spin-orbit coupling is diagonal in the basis of $\sigma_{z}$ eigenstates; therefore the $z$ component of the spin is used as a quantum number below.

The Hamiltonian (2) can be rewritten in a compact form

$$
H_{\mathrm{TB}}=\sum_{k, l} h_{k l} c_{k}^{\dagger} c_{l},
$$

where the elements $h_{k l}$ are defined by Eq. (2). For the eigenfunction $\Psi$ of the atomistic Hamiltonian, with the value of $\Psi_{l}$ on ion $l$, the electron current flowing from ion $l$ to ion $k$, as derived [57] from the Schrödinger equation is

$$
\mathbf{J}_{l j}=\frac{i}{\hbar}\left(h_{l j} \Psi_{l}^{*} \Psi_{j}-h_{j l} \Psi_{l} \Psi_{j}^{*}\right) .
$$

For Hamiltonian eigenstates Eq. (4) provides the probability current flow which is persistent as a characteristic property of a stationary state. The persistent charge current for a given stationary state has the opposite orientation to the probability current. Since the intrinsic spin-orbit coupling is diagonal in the $\sigma_{z}$ spin component, the considered currents are spin polarized in the perpendicular magnetic field. The considered system does not contain short-range scatterers, so the currents are also valley polarized, at least for magnetic fields for which the valley degeneracy is lifted.

\section{B. Continuum Hamiltonian}

The continuum Hamiltonian is used to determine the valley and angular momentum (when available) of the eigenstates calculated in the atomistic approach. The continuum Hamiltonian is a low-energy approximation to the atomistic Hamiltonian. In the low-energy approximation the carriers are described by a spinor wave function with components defined on the $A$ and $B$ sublattices of the silicene crystal lattice $\psi=$ $\left(\begin{array}{ll}\psi_{A} & \psi_{B}\end{array}\right)^{T}$. The low-energy approximation to the atomistic tight-binding Hamiltonian [19] reads

$$
\begin{aligned}
H_{\eta}= & \hbar v_{F}\left(\begin{array}{cc}
0 & k_{x}+\eta i k_{y} \\
k_{x}-\eta i k_{y} & 0
\end{array}\right) \\
& +\left(\begin{array}{cc}
V_{A}(x, y)+\eta \sigma_{z} t_{\mathrm{SO}} & 0 \\
0 & V_{B}(x, y)-\eta \sigma_{z} t_{\mathrm{SO}}
\end{array}\right) \\
& +\frac{g \mu_{B} B}{2} \sigma_{z} I,
\end{aligned}
$$

where $\eta$ stands for the valley index ( $\eta=1$ for the $K$ valley and $\eta=-1$ for the $K^{\prime}$ valley), $I$ is the identity matrix, and $\mathbf{k}=$ $-i \nabla+\frac{e}{\hbar} \mathbf{A}$. In Eq. (5), $v_{F}=3 d t / 2 \hbar$ is the Fermi velocity.

\section{Circular potentials}

For circular potentials $V_{A}(x, y)=V_{A}(r)$ the Hamiltonian eigenfunctions can be labeled by an integer magnetic quantum number $m$,

$$
\Psi_{m, \eta}=\left(\begin{array}{c}
f_{A}(r) \exp (i m \phi) \\
f_{B}(r) \exp \{\exp [i(m-\eta) \phi]\}
\end{array}\right),
$$

where $f_{A}(r)$ and $f_{B}(r)$ are the radial functions on the sublattices. We take a circular flake of radius $R=60 \mathrm{~nm}$. At 


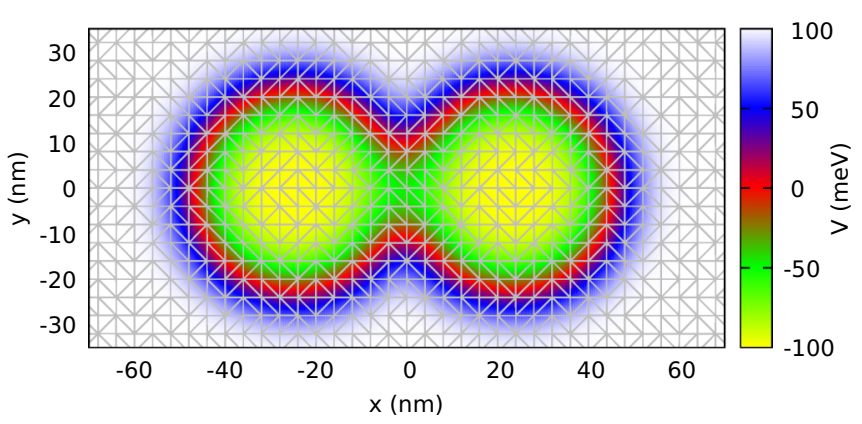

FIG. 2. The electrostatic potential on the $A$ sublattice and a mesh of the triangular elements for the finite-element method (right square equilateral triangles of leg length $5 \mathrm{~nm}$ ) with potential given by Eq. (1) with $l=R=24 \mathrm{~nm}$. The vertical electric field vanishes within the red area. A fragment of the computational box is displayed. In our implementation of the finite-element method the solution in each triangle is spanned in the basis of 6 Lagrange shape functions with the nodes on the corners of the triangle and in the center of each side.

the edge of the flake we apply the zigzag boundary conditions [58]. In order to avoid the fermion-doubling problem we use an asymmetric finite-difference quotient for the first derivative $f^{\prime}=\frac{f(r)-f(r-d r)}{d r}$ instead the symmetric one [58]. The Hamiltonian eigenequation with this quotient can be transformed into a scheme that derives $f_{A}(r-d r)$ and $f_{B}(r-$ $d r)$ from $f_{A}(r)$ and $f_{B}(r)$,

$$
\begin{aligned}
f_{A}(r-d r)= & \frac{d r}{i \hbar v_{F}}\left[E-V_{B}(r)+\eta t_{\mathrm{SO}} \sigma_{z}-\frac{g \mu_{B} B}{2} \sigma_{z}\right] f_{B}(r) \\
& +\left(1+\frac{d r \eta m}{r}+\frac{e B r \eta d r}{2 \hbar}\right) f_{A}(r), \\
f_{B}(r-d r)= & \frac{d r}{i \hbar v_{F}}\left[E-V_{A}(r)-\eta t_{\mathrm{SO}} \sigma_{z}-\frac{g \mu_{B} B}{2} \sigma_{z}\right] f_{A}(r) \\
& +\left(1-\frac{d r \eta(m-\eta)}{r}-\frac{e B r \eta d r}{2 \hbar}\right) f_{B}(r) .
\end{aligned}
$$

The energies of the bound states are determined by the asymptotic condition to be fulfilled at the origin $r=0$, which requires that [58] $f_{A}$ and/or $f_{B}$ function vanish at the origin when $m \neq 0$ and/or $m-\eta \neq 0$, respectively.

\section{Noncircular potentials and a finite-element method}

For coupled systems we perform calculations also for lower symmetry potentials. We take the potential on the $A$ sublattice in the form

$$
V_{A}^{(2)}(\mathbf{r})=V_{g}\left[1-2 e^{-\frac{[\mathbf{r}-(l, 0,0)]^{4}}{R^{4}}}-2 e^{-\frac{[\mathbf{r}+(l, 0,0)]^{4}}{R^{4}}}\right],
$$

where $2 l$ is the distance between the centers of electric field inversion loops. As above, the potential on the $B$ sublattice is taken opposite to the one at the $A$ sublattice. The potential profile on the $A$ sublattice is displayed in Fig. 2. In order to evaluate the eigenstates in the continuum approach we use Hamiltonian (5) in Cartesian coordinates and the finite-element method with the triangular elements on both sublattices and the shape functions in the form of the second-degree Lagrange interpolation polynomials within each of the elements [59]. The elements are right-angle isosceles triangles with a leg length of $5 \mathrm{~nm}$. The side length of a rectangular computational box is taken up to $180 \mathrm{~nm}$. We work with up to 3528 elements.

In order to deal with the fermion-doubling problem and remove the spurious states from the low-energy spectrum [58] we introduce the Wilson term [60] to the Hamiltonian

$$
H_{D}=-W_{D} \nabla^{2} \tau_{z}
$$

with the Wilson parameter $W_{D}=36 \mathrm{meV} \mathrm{nm}^{2}$. This value of the Wilson parameter removes the spurious states with a negligible influence on the actual smooth solutions of the Dirac equation.

\section{RESULTS}

\section{A. Circular potential: Topological confinement}

For the circular topological confinement we take the potential given by Eq. (1) and take $V_{g}=0.1 \mathrm{eV}$ with $R=24 \mathrm{~nm}$. The results are given in Fig. 3. Figure 3(a) shows the results obtained with the atomistic tight-binding approach. The color of the lines corresponds to the localization of the wave function, i.e., the integral of the probability density within the area $r \in(0.6 R, 1.4 R)$. Within the energy gap opened for $E \in\left(-V_{g}, V_{g}\right)$ we find a discrete energy spectrum. All the states within the gap are localized near the zero line.

The energy levels form quadruplets at $B=0$, or more precisely, a pair of doublets split by the spin-orbit interaction of a few meV. The dependence of the energy levels on the magnetic field can be more easily explained using the results of the continuum approach of Fig. 3(b). The energy levels of the discrete part of the spectrum agree very well with the results of the atomistic tight-binding approach [Fig. 3(a)]. The continuum approach explicitly resolves the valley degree of freedom. For the states that are not localized near the zero line, with the energy outside the gap, the results differ, since the energy levels are localized either outside a hexagonal silicene flake (atomistic tight-binding) or near the edges of a circular flake (continuum approach). The form of the boundary condition has no influence on the confined states which are kept off the edge by the electrostatic potential [24].

In Fig. 3(b) we can see that the $K^{\prime}(K)$ energy levels increase (decrease) with increasing $B$. In Figs. 3(d) and 3(e) we display the probability density and probability density current for the first positive-energy spin-down states of the $K$ and $K^{\prime}$ valleys. The results were obtained with the atomistic tight binding; in particular the current distribution was calculated using Eq. (4). The arrows representing the currents show the net currents calculated by summation of interatomic currents on a square mesh of a side length of $2.7 \mathrm{~nm}$. We find that the current orientation depends only on the valley, and that the current circulation in the $K^{\prime}(K)$ valley leaves the negative (positive) potential on the left-hand side of the current orientation in agreement with the nature of the chiral confinement of the zero lines in silicene [33].

For quantitative analysis we define the current moment

$$
\chi=\frac{1}{2} \sum_{k l} \mathbf{r}_{k l} \times\left.\mathbf{J}_{k l}\right|_{z},
$$




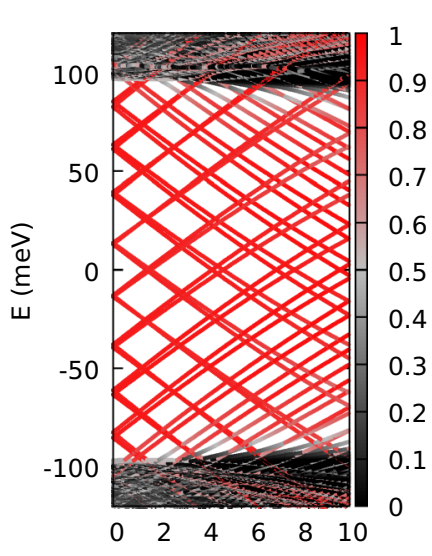

(a)

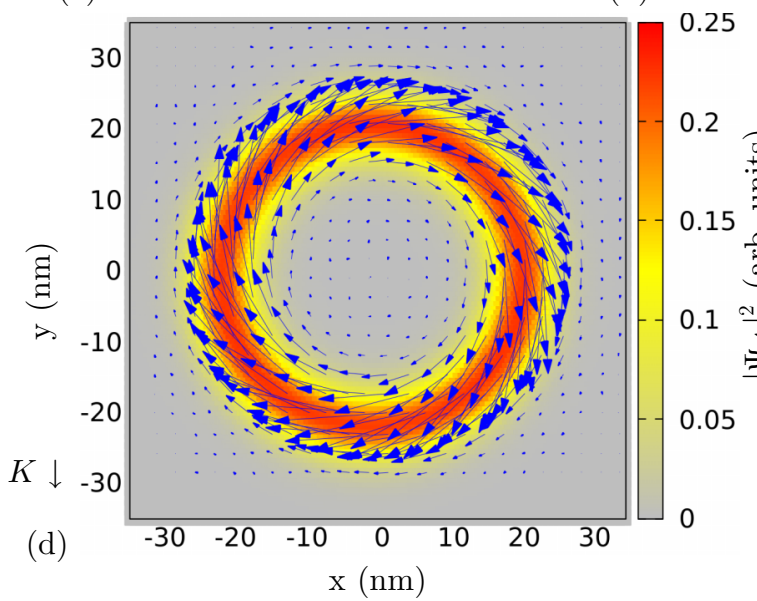

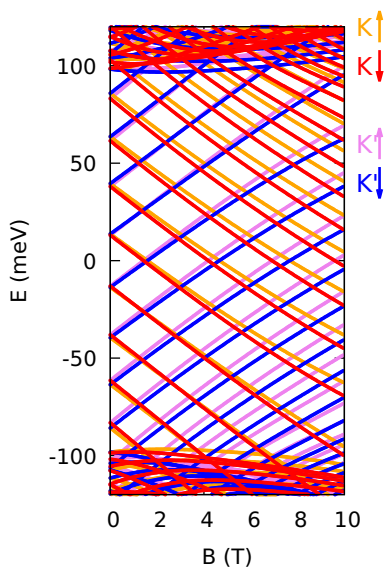

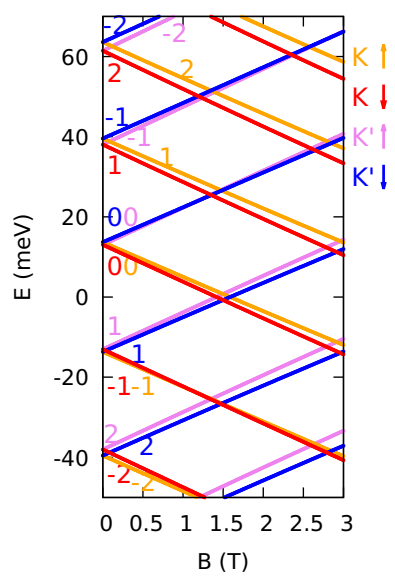

(c)

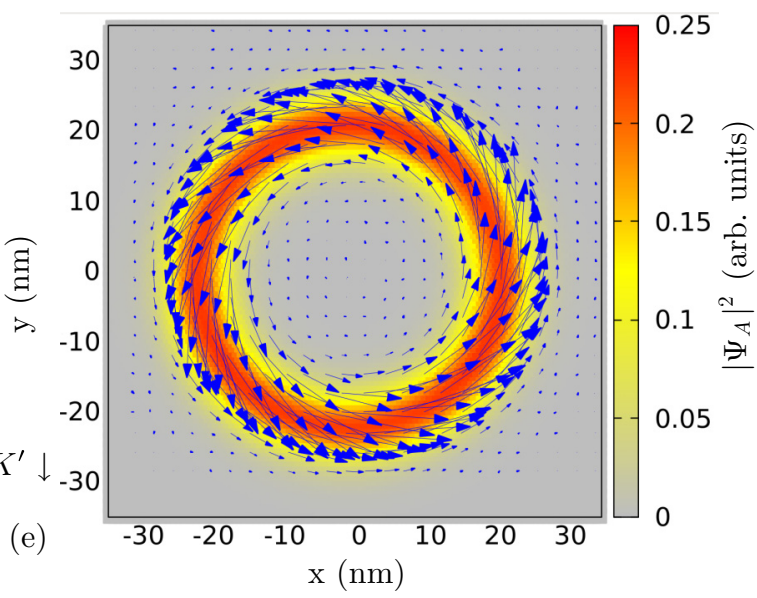

FIG. 3. (a) The energy spectrum as calculated with the atomistic tight-binding approach. Color of the lines shows the localization of the states within the band inversion area calculated by integration of the probability density within the annular area for $r \in(0.6 R, 1.4 R)$. (b) The spectrum as calculated with the finite-difference method. Color of the lines indicates the spin and valley of the states. The states with $|m| \leqslant 16$ are shown. (c) Zoom of (b) with the magnetic quantum numbers $m$ for the $A$ sublattice. Panels (d) and (e) show the probability density and the probability current density for the first $K(\mathrm{~d})$ and $K^{\prime}$ (e) spin-down energy levels at $E>0$.

where $\mathbf{r}_{k l}=\left(\mathbf{r}_{k}+\mathbf{r}_{l}\right) / 2$ is the center of the bond between ion $k$ and ion $l$, and $\mathbf{J}_{k l}$ is the probability current flowing from ion $k$ to ion $l$ as given by Eq. (4). $\chi$ is negative (positive) for clockwise (counterclockwise) probability current flow. The magnetic dipole moment has the opposite orientation to $\chi$.

In Fig. 4(a) we plotted the values of $\chi$ for 20 energy levels of Fig. 3(a) of the lowest absolute value of the energy. We
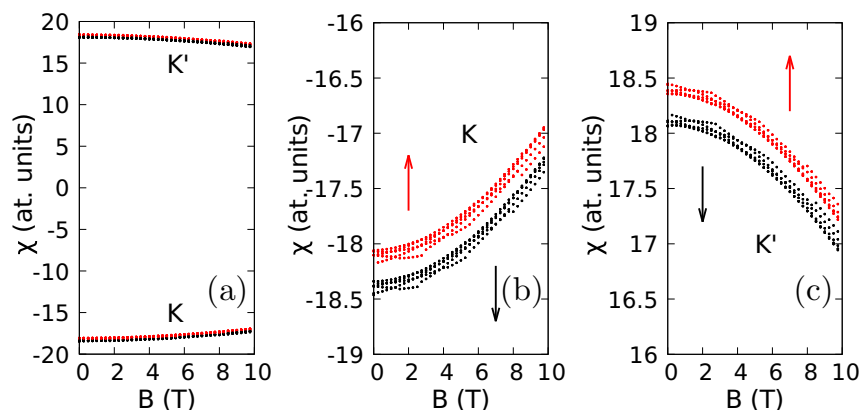

FIG. 4. (a) The current moment as given by Eq. (11) plotted for 20 states of Fig. 3(a) with the smallest absolute values of the energy. The red (black) dots correspond to the spin up (down) states. Panels (b) and (c) show the zoom of the lower (higher) bands of panel (a). can see that the $\chi$ values are nearly the same for all the states of a fixed valley, i.e., the same current orientation, and a very similar distribution of the currents is found for all the localized states of a given valley. In Figs. 4(b) and 4(c) we display the zoom of the parts of Fig. 4(a) that correspond to $K$ and $K^{\prime}$ valley states, respectively. Splitting of the current moment with respect to the spin of the state can be resolved.

The change of the energy levels of Figs. 3(a) and 3(c) with $B$ is consistent with the classical formula for the interaction of the magnetic dipole moment generated by the current loop with the external magnetic field. The counterclockwise probability current $(\chi>0)$ in $K^{\prime}$ states produces a clockwise charge current that generates the magnetic dipole moment oriented to the $-z$ direction, i.e., antiparallel to the external magnetic field, hence the increase of the confined $K^{\prime}$ energy levels with growing $B$. The orientation of the dipole moment and the sign of the energy change is opposite for the $K$ valley.

The structure of energy levels and the angular momentum quantum numbers are presented in Fig. 3(c), which contains a zoom of the continuum spectrum Fig. 3(b) for low absolute value of the energy. We can see that all the energy levels of the degenerate quadruplet have the same value of $|m|$. For the first energy level at the positive energy side the angular momentum 

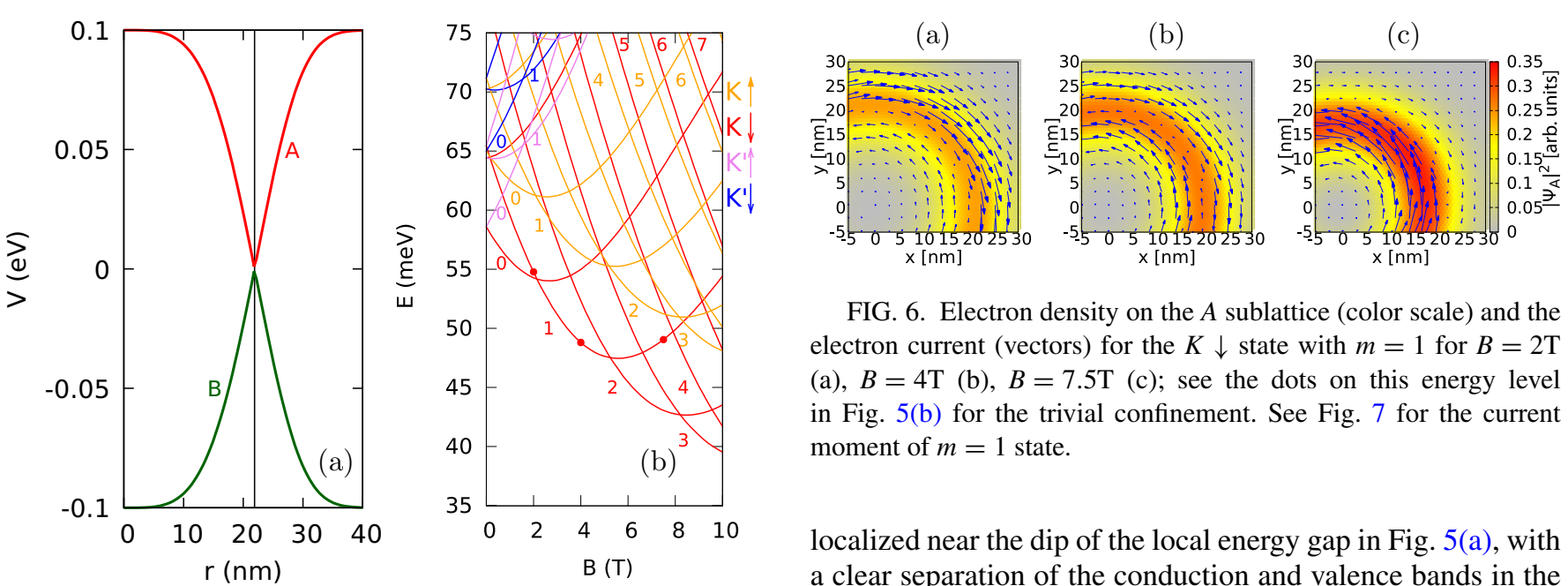

FIG. 6. Electron density on the $A$ sublattice (color scale) and the electron current (vectors) for the $K \downarrow$ state with $m=1$ for $B=2 \mathrm{~T}$ (a), $B=4 \mathrm{~T}$ (b), $B=7.5 \mathrm{~T}$ (c); see the dots on this energy level in Fig. 5(b) for the trivial confinement. See Fig. 7 for the current moment of $m=1$ state.

localized near the dip of the local energy gap in Fig. 5(a), with a clear separation of the conduction and valence bands in the spectrum. In Fig. 5(d) we plot the results of the continuum approach with a zoom of the conduction band side of the spectrum in Fig. 5(b).

For larger $B$ the states near the energy gap [Fig. 5(d)] correspond to $K\left(K^{\prime}\right)$ states at the $E>0(E<0)$ side of the gap. However, there is no general strict correspondence between the valley index and the reaction of the energy level to the change of the magnetic field which is observed for the topological confinement of the preceding subsection. In Fig. 5(b) one can find the localized states which move up and down the energy scale for any valley. Moreover, for a given energy level the sign of the $d E / d B$ derivative changes with $B$; see Fig. 5(c). The sign of this derivative agrees with the current moment as calculated with Eq. (11).

In Fig. 6 the current distribution is plotted for the $K \downarrow m=$ 1 state at $B=2 \mathrm{~T}, 4 \mathrm{~T}$, and $7.5 \mathrm{~T}$ [see the dots in Fig. 5(b)]. In Fig. 7 we plotted the values of the current moment $\chi$ for 30 states of the lowest absolute values of the energy. The larger red, black, and purple dots show the values for the lowestenergy states at the $E>0$ side. The lowest-ones correspond to the $K \downarrow$ states with the values of the $m$ quantum number given in Fig. 7. In Fig. 6 and in Fig. 7 we can see that the

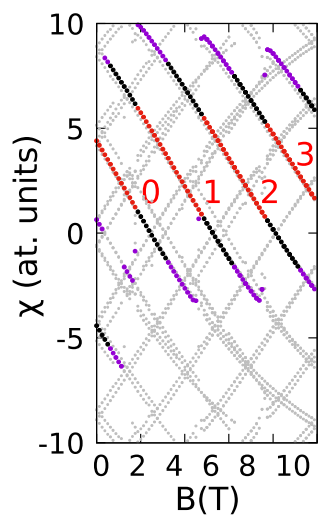

FIG. 7. The current moment as given by Eq. (11) plotted for 30 states of Fig. 5(c) of the smallest absolute values of the energy. With the red, black, and purple dots we mark the lowest, second, and third lowest energy states on the $E>0$ side of the zero energy. By the integers we mark the $m$ values for the lowest-energy $K \downarrow$ states [cf. Fig. 5(b)]. 
(a)

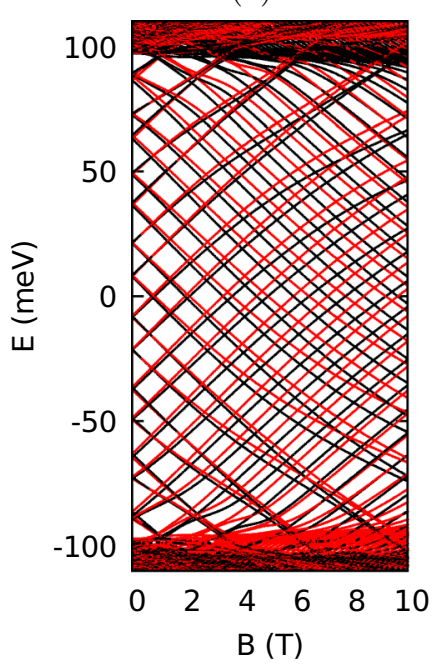

(c)

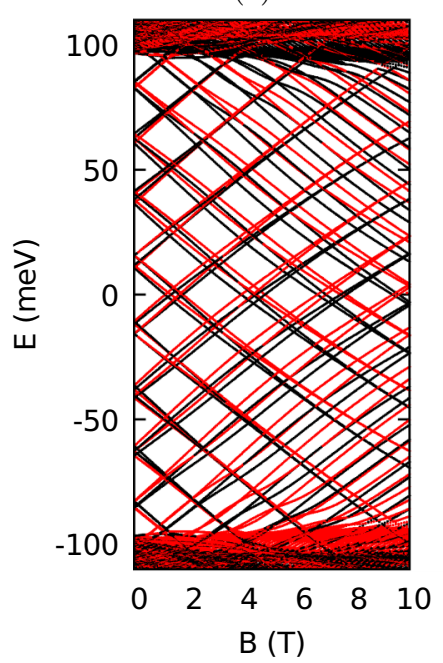

(b)

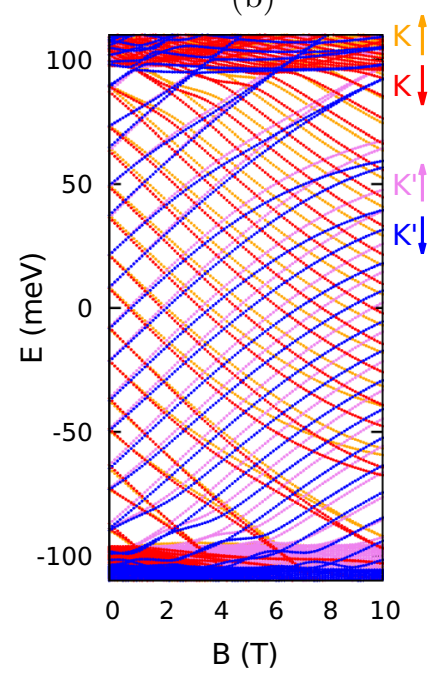

(d)

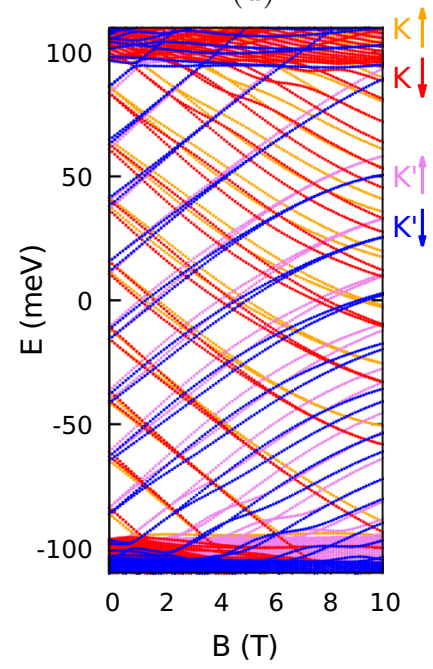

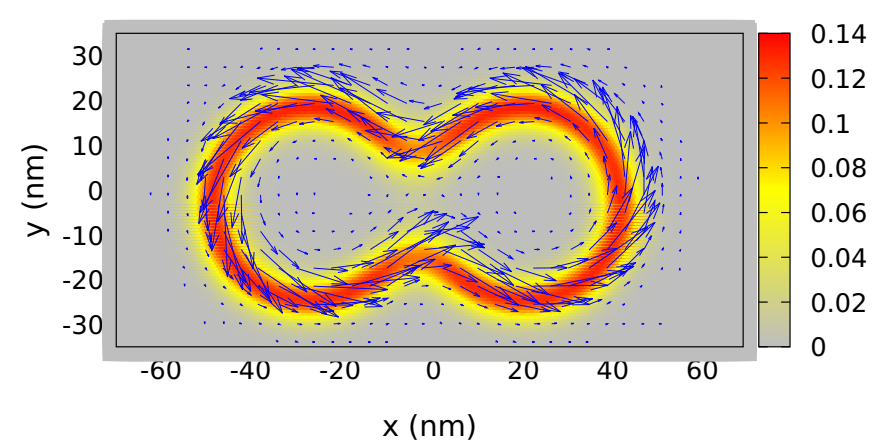

(a)

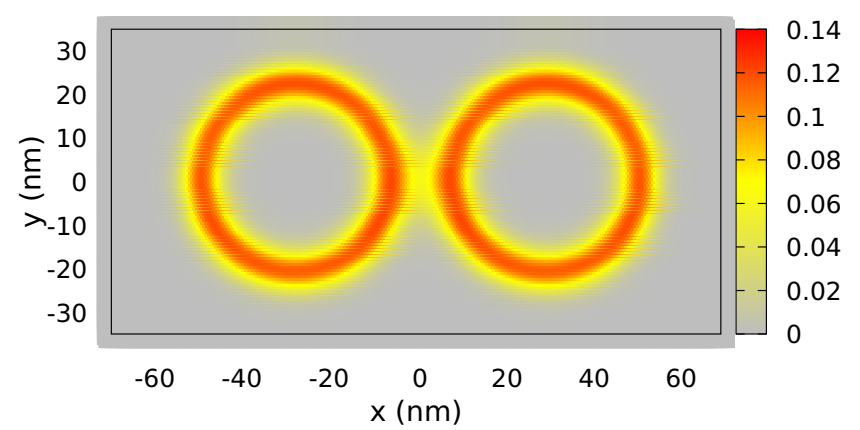

(b)

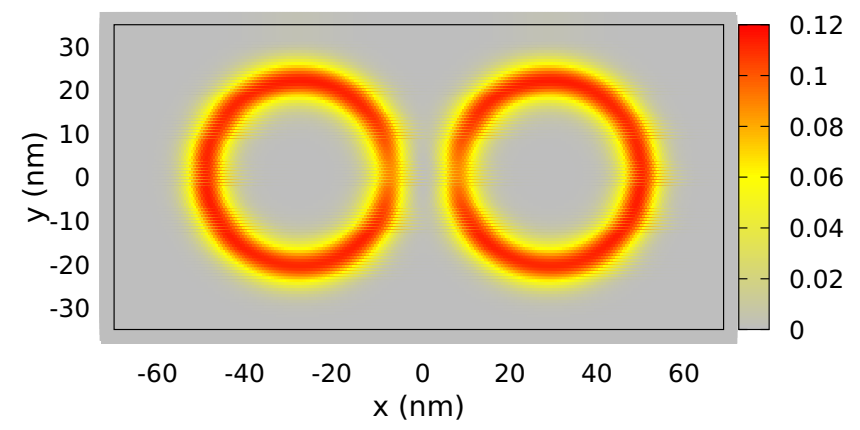

(c)

FIG. 9. (a) Same as Fig. 3(e) but for the double-ring system with $l=R$. (b) The charge density at the $A$ sublattice for the lowest positive energy $K^{\prime} \downarrow$ in the double-ring system with $l=1.2 R$. (c) Same as (b) only for the next higher $K^{\prime} \downarrow$ energy level. The plots (a)-(c) were taken at $B=0$.

symmetry of the system. The persistent current for this ground state appears only induced by external field [50]. For the rings considered here as well as for graphene quantum rings without the Rashba interaction [53] the lowest-positive-energy states at $B=0$ are twofold degenerate with respect valley. Each of the valley degenerate states corresponds to nonzero but opposite persistent current and the magnetic field lifts the degeneracy of the states due to opposite sign of the magnetic dipole moments for these states. According to Ref. [61] the valley crossings in the magnetic field which are quite visible in the spectra for the topological confinement correspond to magnetic fields for which the Berry phase is an integer multiple of $\pi$.

The states studied here for both the trivial and the topological confinement are bound and are similar in this respect to the quantum dots for which the energy levels can be resolved 
(a)

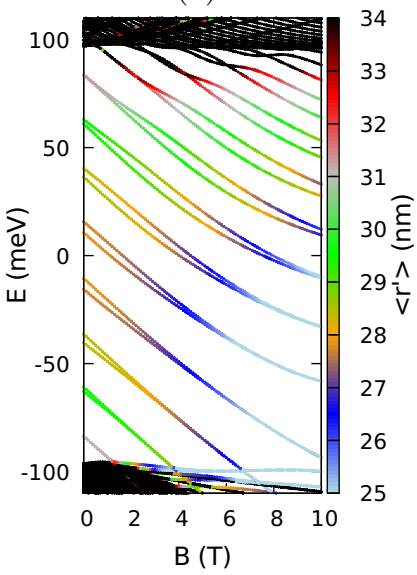

(b)

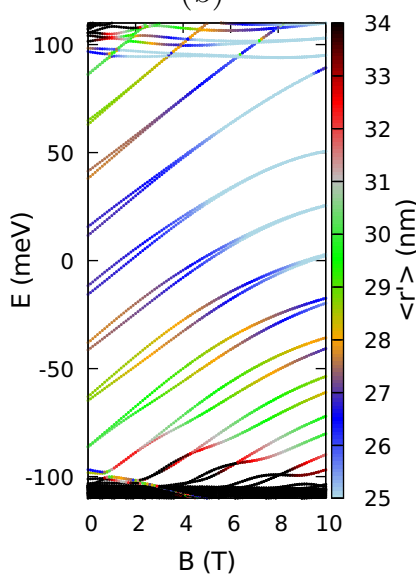

FIG. 10. The energy levels of the $K$ (a) and $K^{\prime}$ (b) energy levels for $l=R$ [taken from Fig. 8(b)] with the color of the lines indicating the average distance to the closer of the two centers of potential given by Eq. (9), $r^{\prime}=\min (|\mathbf{r}-(l, 0,0)|,|\mathbf{r}+(l, 0,0)|)$.

by the transport spectroscopy [1]. The persistent currents can be deduced from the dependence of the energy levels on the external perpendicular magnetic field.

\section{Coupled current loops}

The results of this section are obtained for topological confinement with twin centers given by Eq. (9) (see Fig. 2). The calculated energy spectra are displayed in Fig. 8 for $l=R$ [panels (a) and (b)] and $l=1.2 R$ [panels (c) and (d)]. For $l=R$ the zero line forms a single loop (see Fig. 2). The probability current for the lowest positive energy $K^{\prime} \downarrow$ level at $B=0$ is plotted in Fig. 9(a). The energy spectrum [Figs. 8(a) and 8(b)] resembles the one of the single ring [Figs. 3(a) and 3(b)] only with a larger number of bound energy levels.

For the distance between the ring centers increased to $l=1.2 R$ the energy levels at $B=0$ become nearly twofold degenerate for each spin and valley [Figs. 3(c) and 3(d)]. The rings are nearly separated and a tunnel coupling between them is present [Fig. 9(b)]. The electron density bears signatures of bonding [Fig. 9(b)] and antibonding [Fig. 9(c)] orbitals with enhanced and reduced tunneling between separate rings. Figure 3(d) shows that at higher $B$ the $K^{\prime}$ states at $E>0$ and $K$ energy levels at $E<0$ tend to degenerate in pairs, which indicates lifting of the tunnel coupling between the rings. In order to study this effect in more detail in Figs. 10(a) and 10(b) we plot the spin-down $K$ and $K^{\prime}$ energy levels, respectively, with the color of the lines that indicates the average distance to the nearest center of the ring $\left\langle r^{\prime}\right\rangle$, where for position $\mathbf{r}$ in space $r^{\prime}$ is defined as $r^{\prime}=\min (|\mathbf{r}-(l, 0,0)|,|\mathbf{r}+(l, 0,0)|)$. We can see that for $B=0$ the localization of the wave functions measured with $r^{\prime}$ is the strongest for the states near the zero energy. As the magnetic field grows, the average $r^{\prime}$ is decreased for all the states. For high magnetic field $(B=10 \mathrm{~T})$ the strongest localization is observed for the localized $K$ levels of the lowest energy and for the $K^{\prime}$ energy levels of the highest energy. When $r^{\prime}$ is small the densities are more strongly localized near the centers of separate rings so the tunnel coupling between the rings is lifted and the energy levels become doubly degenerate.

\section{SUMMARY}

We have studied the states bound by inhomogeneous vertical electric field in buckled silicene that is either reduced or inverted along a closed line that supports trivial and topological carrier confinement, respectively. We used the atomistic tightbinding approach and the continuum model for both radially symmetric systems and for pairs of coupled inversion loops. We determined the discrete part of the spectrum within the energy gap that is open by the vertical electric field far from its inversion area. For trivial confinement the orientation of the persistent currents depends on the external magnetic field and can be counterclockwise or clockwise for both valleys. For the topological confinement the orientation of the persistent current is fixed by the valley degree of freedom.

\section{ACKNOWLEDGMENTS}

This work was supported by the National Science Centre (NCN) according to decision DEC-2016/23/B/ST3/00821. The calculations were performed on the PL-Grid Infrastructure and the Prometheus server at ACK Cyfronet AGH.
[1] R. Hanson, L. P. Kouwenhoven, J. R. Petta, S. Tarucha, and L. M. K. Vandersypen, Rev. Mod. Phys. 79, 1217 (2007).

[2] S. Reimann and M. Manninen, Rev. Mod. Phys. 74, 1283 (2002).

[3] J. Gorman, D. G. Hasko, and D. A. Williams, Phys. Rev. Lett. 95, 090502 (2005).

[4] K. D. Petersson, J. R. Petta, H. Lu, and A. C. Gossard, Phys. Rev. Lett. 105, 246804 (2010).

[5] D. D. Awschalom, L. C. Bassett, A. S. Dzurak, E. L. Hu, and J. R. Petta, Science 339,1174 (2013).

[6] M. Eich, R. Pisoni, H. Overweg, A. Kurzmann, Y. Lee, P. Rickhaus, T. Ihn, K. Ensslin, F. Herman, M. Sigrist, K. Watanabe, and T. Taniguchi, Phys. Rev. X 8, 031023 (2018).
[7] M. I. Katsnelson, K. S. Novoselov, and A. K. Geim, Nat. Phys. 2, 620 (2006).

[8] E. McCann, Phys. Rev. B 74, 161403(R) (2006).

[9] E. V. Castro, K. S. Novoselov, S. V. Morozov, N. M. R. Peres, J. M. B. Lopes dos Santos, J. Nilsson, F. Guinea, A. K. Geim, and A. H. Castro Neto, Phys. Rev. Lett. 99, 216802 (2007).

[10] J. B. Oostinga, H. B. Heersche, X. Liu, A. F. Morpurgo, and L. M. K. Vandersypen, Nat. Mater. 7, 151 (2008).

[11] F. Xia, D. B. Farmer, Y. M. Lin, and P. Avouris, Nano Lett. 10, 715 (2010).

[12] T. Ohta, A. Bostwick, T. Seyller, K. Horn, and E. Rotenberg, Science 313, 951 (2006).

[13] Z. Ni, Q. Liu, K. Tang, J. Zheng, J. Zhou, R. Qin, Z. Gao, D. Yu, and J. Lu, Nano Lett. 12, 113 (2012). 
[14] N. D. Drummond, V. Zolyomi, and V. I. Fal'ko, Phys. Rev. B 85, 075423 (2012).

[15] A. Molle, J. Goldberger, M. Houssa, Y. Xu, S.-C. Zhang, and D. Akinwande, Nat. Mater. 16, 163 (2017).

[16] M. Ezawa, E. Salomon, P. De Padova, D. Solonenko, P. Vogt, M. E. Davila, A. Molle, T. Angot, and G. Le Lay, Riv. Nuovo Cimento 41, 175 (2018).

[17] S. Chowdhury and D. Jana, Rep. Prog. Phys. 79, 126501 (2016).

[18] M. Ezawa, Phys. Rev. Lett. 109, 055502 (2012).

[19] C.-C. Liu, W. Feng, and Y. Yao, Phys. Rev. Lett. 107, 076802 (2011); C.-C. Liu, J. Jiang, and Y. Yao, Phys. Rev. B 84, 195430 (2011).

[20] J. M. Pereira, Jr., P. Vasilopoulos, and F. M. Peeters, Nano Lett. 7, 946 (2007).

[21] A. M. Goossens, S. C. M. Driessen, T. A. Baart, K. Watanabe, T. Taniguchi, and L. M. K. Vandersypen, Nano Lett. 12, 4656 (2012).

[22] M. T. Allen, J. Martin, and A. Yacoby, Nat. Commun. 3, 934 (2012).

[23] D. P. Żebrowski, E. Wach, and B. Szafran, Phys. Rev. B 88, 165405 (2013).

[24] B. Szafran, D. Zebrowski, and A. Mreńca-Kolasińska, Sci. Rep. 8, 7166 (2018).

[25] I. Martin, Y. M. Blanter, and A. F. Morpurgo, Phys. Rev. Lett. 100, 036804 (2008).

[26] Z. Qiao, J. Jung, Q. Niu, and A. H. MacDonald, Nano Lett. 11, 3453 (2011).

[27] M. Ezawa, New J. Phys. 14, 033003 (2012).

[28] S.-G. Cheng, H. Liu, H. Jiang, Q.-F. Sun, and X. C. Xie, Phys. Rev. Lett. 121, 156801 (2018).

[29] L. Ju, Z. Shi, N. Nair, Y. Lv, C. Jin, J. Velasco, Jr., C. OjedaAristizabal, H. A. Bechtel, M. C. Martin, A. Zettl, J. Analytis, and F. Wang, Nature (London) 520, 650 (2015).

[30] A. Vaezi, Y. Liang, D. H. Ngai, L. Yang, and E.-A. Kim, Phys. Rev. X 3, 021018 (2013).

[31] R. Bistritzer and A. H. MacDonald, Proc. Natl. Acad. Sci. USA 108, 12233 (2011).

[32] S. G. Xu, A. I. Berdyugin, P. Kumaravadivel, F. Guinea, R. Krishna Kumar, D. A. Bandurin, S. V. Morozov, W. Kuang, B. Tsim, S. Liu, J. H. Edgar, I. V. Grigorieva, V. I. Fal'ko, M. Kim, and A. K. Geim, Nat. Commun. 10, 4008 (2019).

[33] B. Szafran, B. Rzeszotarski, and A. Mreńca-Kolasińska, Phys. Rev. B 100, 085306 (2019).

[34] M. Z. Hasan and C. L. Kane, Rev. Mod. Phys. 82, 3045 (2010).

[35] J. H. Bardarson and J. E. Moore, Rep. Prog. Phys. 76, 056501 (2013).

[36] X.-L. Qi and S.-C. Zhang, Rev. Mod. Phys. 83, 1057 (2011).

[37] D. A. Abanin and L. S. Levitov, Science 317, 641 (2007); J. R. Williams, L. DiCarlo, and C. M. Marcus, ibid. 317, 638 (2007).

[38] T. Taychatanapat, J. Y. Tan, Y. Yeo, K. Watanabe, T. Taniguchi, and B. Iezyilmaz, Nat. Commun. 6, 6093 (2015).
[39] P. Rickhaus, P. Makk, M.-H. Liu, E. Tovari, M. Weiss, R. Maurand, K. Richter, and C. Schoenenberger, Nat. Commun. 6, 6470 (2015).

[40] J. R. Williams and C. M. Marcus, Phys. Rev. Lett. 107, 046602 (2011).

[41] A. Cresti, G. Grosso, and G. P. Parravicini, Phys. Rev. B 77, 115408 (2008).

[42] S. P. Milovanovic, M. Ramezani Masir, and F. M. Peeters, Appl. Phys. Lett. 105, 123507 (2014).

[43] K. Kolasiński, A. Mreńca-Kolasińska, and B. Szafran, Phys. Rev. B 95, 045304 (2017).

[44] S. Viefers, P. Koskinen, P. Singha Deo, and M. Manninen, Phys. E (Amsterdam) 21, 1 (2004).

[45] V. M. Fomin, Quantum ring: A unique playground for the quantum-mechanical paradigm, Physics of Quantum Rings (Springer International Publishing, Cham, 2018).

[46] M. Büttiker, Y. Imry, and R. Landauer, Phys. Lett. A 96, 365 (1983).

[47] H.-F. Cheung, Y. Gefen, E. K. Riedel, and W.-H. Shih, Phys. Rev. B 37, 6050 (1988).

[48] N. A. J. M. Kleemans, I. M. A. Bominaar-Silkens, V. M. Fomin, V. N. Gladilin, D. Granados, A. G. Taboada, J. M. Garcia, P. Offermans, U. Zeitler, P. C. M. Christianen, J. C. Maan, J. T. Devreese, and P. M. Koenraad, Phys. Rev. Lett. 99, 146808 (2007).

[49] Q.-f. Sun, X. C. Xie, and J. Wang, Phys. Rev. Lett. 98, 196801 (2007); Q.-F. Sun, X. C. Xie, and J. Wang, Phys. Rev. B 77, 035327 (2008).

[50] B. Szafran, Phys. Rev. B 77, 205313 (2008).

[51] P. Recher, B. Trauzettel, A. Rycerz, Y. M. Blanter, C. W. J. Beenakker, and A. F. Morpurgo, Phys. Rev. B 76, 235404 (2007).

[52] L. Ying and Y.-C. Lai, Phys. Rev. B 96, 165407 (2017).

[53] N. Bolivar, E. Medina, and B. Berche, Phys. Rev. B 89, 125413 (2014).

[54] D. Faria, A. Latge, S. E. Ulloa, and N. Sandler, Phys. Rev. B 87, 241403(R) (2013).

[55] W. G. van der Wiel, S. De Franceschi, J. M. Elzerman, T. Fujisawa, S. Tarucha, and L. P. Kouwenhoven, Rev. Mod. Phys. 75, 1 (2002).

[56] C. L. Kane and E. J. Mele, Phys. Rev. Lett. 95, 226801 (2005).

[57] K. Wakabayashi, Phys. Rev. B 64, 125428 (2001).

[58] B. Szafran, A. Mreńca-Kolasińska, and D. Żebrowski, Phys. Rev. B 99, 195406 (2019).

[59] P. Solin, Partial Differential Equations and the Finite Element Method (John Wiley and Sons, Inc., New York, 2005).

[60] Y. Tanimura, K. Hagino, H. Z. Liang, Y. Tanimura, K. Hagino, and H. Z. Liang, Prog. Theor. Exp. Phys. 2015, 073D01 (2015).

[61] Z. Hou, Y.-F. Zhou, X. C. Xie, and Q.-F. Sun, Phys. Rev. B 99, 125422 (2019). 